\title{
Calculation of Effective Permeability in Fractured Porous Media Using Finite Volume Method
}

\author{
$\underline{\text { Chen Gou }}^{\text {a }}$, N. Natarajan ${ }^{\mathrm{b}}$, Zhao Feng Tian ${ }^{\mathrm{a}}$, Chaoshui Xu ${ }^{\mathrm{b}}$ \\ ${ }^{a}$ School of Mechanical Engineering, the University of Adelaide, Adelaide, SA5005, Australia \\ ${ }^{b}$ School of Civil, Environmental and Mining Engineering, the University of Adelaide, SA 5005, Australia \\ Email: Zhao.tian@adelaide.edu.au
}

\begin{abstract}
This paper focuses on calculation of effective permeability in fractured porous media with blocks matrix using finite volume Computational Fluid Dynamics (CFD) method. This fractured media with blocks matrix can be found in the Enhanced Geothermal Systems (EGS). A fracture network consists of nine square $(3 * 3)$ rock blocks and the fractures between the rocks. Periodic boundaries are used for four side of the domain, while zero mass flow rate is implemented in the vertical direction and a mass flow rate of 0.0092 $\mathrm{kg} / \mathrm{s}$ is implemented in the horizontal direction.

Effects of rock material permeability of the rock blocks on the effective permeability of the whole domain are investigated. Five cases with different rock permeability, namely, $0 \mathrm{~m}^{2}, 0.1 \mathrm{E}-7 \mathrm{~m}^{2}, 0.1 \mathrm{E}-6 \mathrm{~m}^{2}, 0.1 \mathrm{E}-5 \mathrm{~m}^{2}$, and $0.1 \mathrm{E}-5 \mathrm{~m}^{2}$, are calculated based on the CFD model. It is found that the effective permeability $K_{x}$ increases significantly as the increase of the rock permeability, indicating the importance of taking into consideration of rock permeability in calculating effective permeability of EGS systems.
\end{abstract}

Keywords: Geothermal System, CFD, fracture, Pressure drop, effective permeability 


\section{INTRODUCTION}

A vast amount of thermal energy is stored in the earth crust. And the thermal energy can be exploited to generate electrical power by the Enhanced Geothermal System (EGS). One kind of EGS that has large potential use in Australia is the Hot dry rock (HDR) system. For an EGS in HDR, two vertical wells can be drilled from ground into the HDR area that is at a high temperature (say $400{ }^{\circ} \mathrm{C}$ ), then high pressure fluid flow can be injected into the fracture of HDR from one vertical well that is called injection well; the fluid flowing through the fractures gained heat from the surrounding HDR (Chopra \& Wyborn, 2003). The high temperature water from the HDR factures can be extracted through other well(s) called product well(s) and then can be used for generation of electricity.

For HDR, the channel walls contain cracks and fissures because of the initial hydraulic fracturing process. Hence, the permeable characteristics of the boundary will influence the fluid flow in the fracture. Since the rocks are porous the fluid flowing in the fracture will diffuse into the pores of the rock matrix (Kosakowski \& Berkowitz, 1999). The amount of fluid flow diffusing into the rocks depends on the porosity and permeability of the rocks. More specifically, if the rock matrix has a large value of porosity and permeability, it will cause more fluid loss, because more water would diffuse into the pores of the rocks. By contrast, the small values of porosity and permeability would mean less fluid loss. Hence, the porosity and permeability have great impact on the efficiency of EGS. Moreover, the porosity and permeability in the rocks will also determine the effective permeability of large rock blocks. This effective permeability can be used to compute the total mass flow rate and heat flux in an EGS and the heat flux. Therefore, it is essential to find the relationship between the effective permeability of the large scale blocks and the absolute permeability of fractures and the rocks.

This project simulates fluid flows within a fractured porous media in an EGS using the computational fluid dynamics (CFD) techniques and analyses the pressure drop of fluid flow in the fracture. Based on the pressure drop, the effective permeability of the fractured porous media is obtained. Figure 1 below shows the physical system considered for this study.

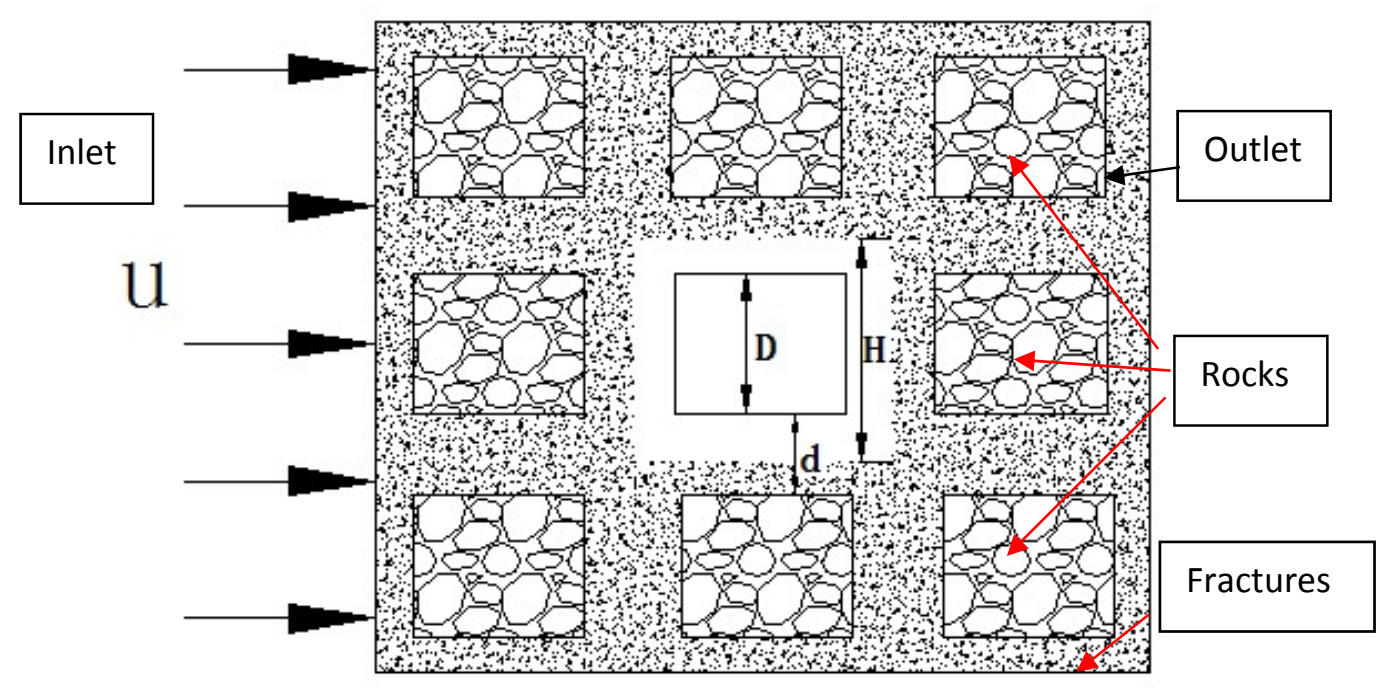

Figure 1 Fluid flow in fractured porous media

In Figure 1, the square blocks represent rocks, while the passages between them are the fractures filled with porous media. The present geometry accounts for both horizontal and vertical fractures, and the water flows in the domain. In addition, the fluid flow is assumed to follow periodic boundary conditions. Some researchers, including Liu et al. (2009), have calculated the effective permeability in a similar geometry. However, they have only obtained the effective permeability for the cases that the rocks are impermeable solid. This study further the study taking the rocks as porous medium material with different absolute permeability. In this project, fluid flow through the fractured porous medium has been simulated using to obtain pressure drops in the domain, which is used to calculate the effective horizontal permeability and vertical permeability. 


\section{CFD METHODS}

The CFD domain includes into fractured domain and blocks domain as shown in Figure 1. The model (45 $\mathrm{cm}^{*} 45 \mathrm{~cm}$ ) consist of nine square $(3 * 3)$ blocks of size $\mathrm{D}=10 \mathrm{~cm}$ and the passages between them are the fractures of aperture $d=5 \mathrm{~cm}$ filled with porous media. The flow is two dimensional (2D). The geometry was generated by using ANSYS/Designmodeler. Three meshes are generated by using ANSYS/Meshing with different elements and finally the mesh of 516677 elements is used for this paper.

The fluid flow is laminar and there is no heat transfer. As the rock matrix has different absolute permeability and porosity, the porosity and absolute permeability of fractured porous media are constants with values of 0.5 and $0.1 \mathrm{E}-4 \mathrm{~m}^{2}$, respectively. The vertical and horizontal fractures constitute the porous domain, the top and bottom sides are symmetric walls. The inlet and outlet are set as periodic boundaries with specified mass flow rate. The mass flow rate is $0.0092 \mathrm{~kg} / \mathrm{s}$.

\section{RESULTS AND DISCUSSIONS}

In this model, the fluid flow has two directions, horizontal direction and vertical direction. Based on the direction permeability method reported in Renard and De Marsily (1997), the effective permeability along the horizontal direction can be calculated by the equations below:

$$
K_{x}=-v \frac{Q_{x}}{S_{x}} \frac{L_{x}}{\Delta p_{x}}
$$

Where, $K_{x}$ is the effective permeability in x (horizontal) direction. $v$ is kinematic viscosity of water and $Q_{x}$ is the mass flow rate in $\mathrm{x}$ (horizontal) direction. $L_{x}$ is the distance between two sides and $S_{x}$ is the cross section area in horizontal direction. $\Delta P_{x}$ is the pressure drop between the two periodic sides in horizontal direction.

In this study the top and bottom boundaries of the flow domain are taken as periodic boundaries without mass flow rate. The pressure gradient and flow velocity along vertical y-axis are therefore zero. The porosity and permeability of fractured porous media remaining stable, while the porosity and permeability of blocks are changed and the pressure drops between the two periodic sides in horizontal direction can be obtained. The results are as follow:

Table 1 Predicted $K_{x}$ values for different cases

\begin{tabular}{|c|c|c|c|c|}
\hline Case number & Rocks porosity & Rocks Permeability $\left(\mathrm{m}^{2}\right)$ & Pressure drop(pa) & $K_{x}\left(\mathrm{~m}^{2}\right)$ \\
\hline 1 & & Solid & 0.142 & 2.89E-05 \\
\hline 2 & 0.2 & $0.1 \mathrm{E}-7$ & 0.089 & 4.61E-05 \\
\hline 3 & 0.2 & $0.1 \mathrm{E}-6$ & 0.074 & $5.48 \mathrm{E}-05$ \\
\hline 4 & 0.2 & $0.1 \mathrm{E}-5$ & 0.057 & $7.16 \mathrm{E}-05$ \\
\hline 5 & 0.2 & $0.1 \mathrm{E}-4$ & 0.007 & $5.55 \mathrm{E}-04$ \\
\hline
\end{tabular}

In Case 1, the rocks are solid blocks with neither porosity nor permeability. The pressure drop in the whole domain in $x$ direction is $0.142 \mathrm{pa}$. The calculated effective permeability is $2.89 \mathrm{E}-05 \mathrm{~m}^{2}$. In Case 2 , when the porosity of rocks is set to be 0.2 and the rock permeability is $0.1 \mathrm{E}-7 \mathrm{~m}^{2}$. The pressure drop within the domain reduces to be $0.057 \mathrm{pa}$ and the calculated $K_{x}$ increases to $4.61 \mathrm{E}-5 \mathrm{~m}^{2}$. When further increasing the rock permeability to $0.1 \mathrm{E}-6 \mathrm{~m}^{2}$, the effective permeability increases to $5.48 \mathrm{E}-05 \mathrm{~m}^{2}$. In Case 5 , when the rock permeability is increased to $0.1 \mathrm{E}-4 \mathrm{~m}^{2}, K_{x}$ increases to $5.55 \mathrm{E}-5 \mathrm{~m}^{2}$, almost double of that of the impermeable case. It is found that the effective permeability $K_{x}$ increases significantly as the increase of the rock permeability. 
Gou, Natarajan, Tian, and Xu, Calculation of effective permeability for fracture porous media

The developed procedure to calculate effective permeability can be later used to calculate the effective permeability of large subdomains in HDR systems. The detailed structure of the fracture in a subdomain can be generated by different fracture models (Xu \& Dowd, 2010, Xu, Dowd, Mardia, \& FOWELL, 2006).

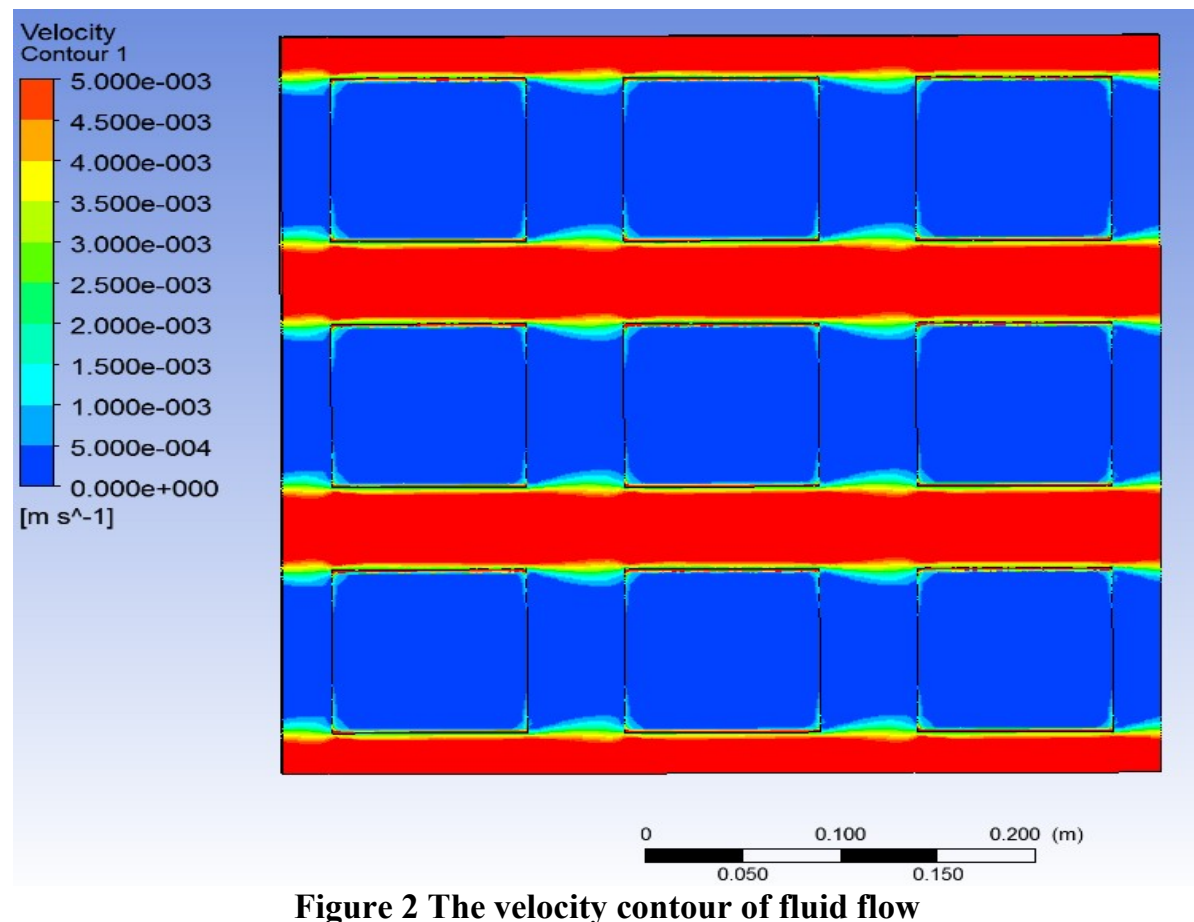

The flow fields are further investigated. As shown in Figure 2 for Case 2, velocity of fluid flow in fractures is much larger compared to the velocity in the rock blocks. This is because the permeability of the rocks is lower than that of the fracture. The flow shows a periodic pattern in the domain. As observed in Figure 3, the pressure is high at the inlet, and gradually decreases across the domain. The pressure distribution at the outlet is almost uniform.

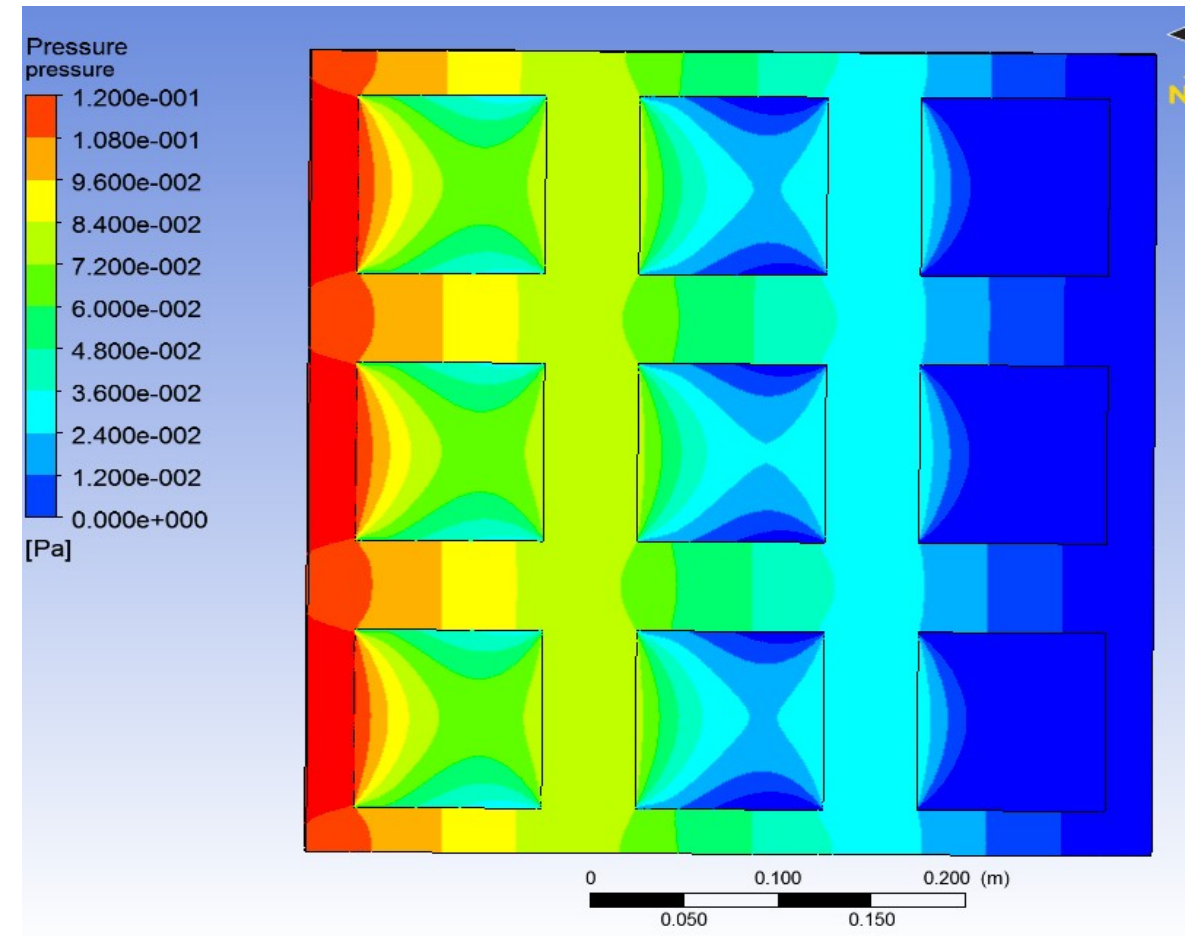

Figure 3 The pressure distribution of fluid flow 


\section{CONCLUSION}

This paper focuses on applying CFD tools to predict the fluid flows and pressure drops in a fractured porous medium, which consists of rocks and fractures. Periodic boundaries are used in the sides of the domain. The aim is to predict the pressure drops of fluid flow within the domain, and thereby to calculate the effective permeability of the whole domain. The calculated effective permeability can then be used to calculate the mass flow rate and heat flux in the EGS.

The procedure of the calculation of effective permeability is reported in the paper. First time in the literature, the effects of rock permeability on the effective permeability are investigated in this particular domain. It is found that the effective permeability $K_{x}$ increases significantly as the increase of the rock permeability, indicating the importance of taking into consideration of permeability of rocks in calculating effective permeability of EGS systems in the future.

\section{ACKNOWLEDGEMENTS}

The work described here was partially funded by Australian Research Council Discovery Project grant DP110104766 and South Australian Centre for Geothermal Energy Research, University of Adelaide.

\section{REFERENCES}

Chopra, P., \& Wyborn, D., (2003). Australia's first hot dry rock geothermal energy extraction project is up and running in granite beneath the Cooper Basin, NE South Australia. In Magmas to Mineralisation: The Ishihara Symposium. Geoscience Australia, Record , 14, 43-45.

Kosakowski, G., \& Berkowitz, B., (1999). Flow pattern variability in natural fracture intersections. Geophysical Research Letters, 26(12), 1765-1768.

Liu, J.J. , Sano, Y., \& Nakayama A., (2009). A simple mathematical model for determining the equivalent permeability of fractured porous media. Communications in Heat and Mass Transfer, 36, 220-224.

Renard, P., \& De Marsily, G. (1997). Calculating effective permeability: a review. Advances In Water Resources, 20(5), 253-278.

Xu, C., \& Dowd, P.A. (2010). A new computer code for discrete fracture network modelling. Computers \& Geosciences, 36(3), 292-301.

Xu, C., Dowd, P. A., Mardia, K. V., \& Fowell, R. J. (2006). A connectivity index for discrete fracture networks. Mathematical Geology, 38(5), 611-634. 